\title{
Odor Identification in Rats: Behavioral and Electrophysiological Evidence of Learned Olfactory-Auditory Associations
}

\author{
(D) Jonas K. Olofsson, ${ }^{1,2,3}$ Guangyu Zhou, ${ }^{4}$ Brett S. East, ${ }^{1,2}$ Christina Zelano, ${ }^{4}$ and ${ }^{\circledR}$ Donald A. Wilson ${ }^{1,2,5}$
}

\author{
https://doi.org/10.1523/ENEURO.0102-19.2019
}

\begin{abstract}
${ }^{1}$ Emotional Brain Institute, Nathan S. Kline Institute, Orangeburg, NY 10962, ${ }^{2}$ Department of Child and Adolescent Psychiatry, New York University School of Medicine, New York, NY 10016, ${ }^{3}$ Department of Psychology, Stockholm University, Stockholm SE-10691, Sweden, ${ }^{4}$ Department of Neurology, Northwestern University Feinberg School of Medicine, Chicago, IL 60611, and ${ }^{5}$ Department of Neuroscience and Physiology, New York University School of Medicine, New York, NY 10016
\end{abstract}

\begin{abstract}
The ability to recognize and identify a smell is highly dependent on multisensory context and expectation, for example, hearing the name of the odor source. Here, we develop a novel auditory-odor association task in rats, wherein the animal learns that a specific auditory tone, when associated with a specific odor, predicts reward (Go signal), whereas the same tone associated with a different odor, or vice versa, is not (No-Go signal). The tone occurs prior to the onset of the odor, allowing physiological analyses of sensory-evoked local field potential (LFP) activity to each stimulus in primary auditory cortex and anterior piriform cortex (aPCX). In trained animals that have acquired the task, both auditory and subsequent olfactory cues activate $\beta$ band oscillations in both the auditory cortex and PCX, suggesting multisensory integration. Naive animals show no such multisensory responses, suggesting the response is learned. In addition to the learned multisensory evoked responses, functional connectivity between auditory cortex and PCX, as assessed with spectral coherence and phase lag index (PLI), is enhanced. Importantly, both the multi-sensory evoked responses and the functional connectivity are contextdependent. In trained animals, the same auditory stimuli presented in the home cage evoke no responses in auditory cortex or PCX, and functional connectivity between the sensory cortices is reduced. Together, the results demonstrate how learning and context shape the expression of multisensory cortical processing. Given that odor identification impairment is associated with preclinical dementia in humans, the mechanisms suggested here may help develop experimental models to assess effects of neuropathology on behavior.
\end{abstract}

Key words: auditory perception; electrophysiology; learning; olfaction; sensation

\section{Significance Statement}

An important feature in mammalian olfaction is the multisensory support provided by "higher" senses, such as hearing and vision. In humans, such multisensory context and expectation, for example, hearing the name of the odor source, facilitates the identification of a smell. An impaired ability to identify odors is a sensitive predictor of cognitive decline and neurodegenerative dementia. We found that rats trained on a tone-odor association task, but not untrained rats, showed elevated electrophysiological responses in both auditory and olfactory cortices, as well as increased functional connectivity between these regions, during task engagement. These results provide evidence of a multisensory integration process that might provide clues to how neuropathology affects the brain. 


\section{Introduction}

Multisensory processing allows activity in one sensory pathway to modulate activity in another, in turn shaping perception. For example, in the McGurk effect, discordant visual cues influence auditory perception to the point where a subject hears a syllable consistent with the visual mouth movements of the speaker instead of the actual auditory syllable (McGurk and MacDonald, 1976). The convergence of sensory processing streams that enables multisensory perception has been described in increasingly earlier stages of "unimodal" sensory pathways, including classic primary sensory areas (Calvert et al., 1999; Foxe et al., 2000). Importantly, more recent evidence suggests that multisensory integration can be contextdependent, and not a stable result of fixed anatomic convergence (Schroeder et al., 2001; Choi et al., 2018).

The vast majority of work on multisensory processing has occurred in classic thalamocortical sensory systems such as vision and audition. However, multisensory integration also occurs in the olfactory system (Gottfried and Dolan, 2003; Wesson and Wilson, 2010; Cohen et al., 2011; Mandairon et al., 2014; Karunanayaka et al., 2015; Boisselier et al., 2018), an evolutionarily older system with no thalamic relay between the periphery and the olfactory cortex, which itself is a three-layered archicortex (Neville and Haberly, 2004; Wilson and Sullivan, 2011; Bekkers and Suzuki, 2013). For example, visual cues can influence olfactory perception, e.g., red liquids with strawberry scent smell more intense than clear liquids (Zellner and Kautz, 1990; Morrot et al., 2001; Demattè et al., 2009), as can gustatory, somatosensory and auditory cues (Cain, 1979; Dalton et al., 2000; Stevenson and Mahmut, 2011; Stevenson et al., 2012). Much of the multisensory influences on olfactory perception are related to semantic associations of the odor (i.e., identifiability or familiarity; de Araujo et al., 2005; Stevenson et al., 2012), nonetheless, multisensory stimulation is associated with modulation of odor-evoked activity in central olfactory and limbic regions in humans (Gottfried and Dolan, 2003; de Araujo et al., 2005; Karunanayaka et al., 2015; Zhou et al., 2019) and animal models (Kadohisa et al., 2005; Wesson and

Received March 18, 2019; accepted July 15, 2019; First published July 30, 2019.

The authors declare no competing financial interests.

Author contributions: J.K.O. and D.A.W. designed research; J.K.O., B.S.E., and D.A.W. performed research; J.K.O., G.Z., B.S.E., C.Z., and D.A.W. wrote the paper; G.Z., C.Z., and D.A.W. analyzed data.

This work was supported by a Knut and Alice Wallenberg Foundation Grant 2016:0229; a Pro Futura Scientia Fellowship (J.K.O.); and United States Department of Health and Human Services (HHS) National Institutes of Health (NIH) National Institute on Deafness and Other Communication Disorders (NIDCD) Grants R01-DC003906 (to D.A.W.), F32-DC017356-01 (to B.S.E.), and R01-DC016364 (to C.Z.).

Acknowledgements: We thank Emmanuelle Courtiol, Gloria Fleming and Catia Teixeira for helpful advice and assistance.

Correspondence should be addressed to Donald A. Wilson at donald.wilson@nyumc.org.

https://doi.org/10.1523/ENEURO.0102-19.2019

Copyright (C) 2019 Olofsson et al.

This is an open-access article distributed under the terms of the Creative Commons Attribution 4.0 International license, which permits unrestricted use, distribution and reproduction in any medium provided that the original work is properly attributed.
Wilson, 2010; Mandairon et al., 2014; Maier et al., 2015; Veyrac et al., 2015).

A vulnerability in links between the olfactory system and other multisensory and language related regions has been hypothesized to underlie the sensitivity of odor identification to a variety of disorders including mild cognitive impairment, Alzheimer's disease and primary progressive aphasia (Devanand et al., 2008; Olofsson and Gottfried, 2015; Olofsson et al., 2009, 2013; MacDonald et al., 2018). However, recent evidence suggests that an auditory word cue that primes a matching odor may generate a strong functional coupling between primary olfactory cortex and higher order auditory cortex in humans (Zhou et al., 2019). Specifically, subjects prepared for intracranial electroencephalography were presented with an auditory word cue of an odor name followed by a matching or non-matching odor. Following the auditory word, but before delivery of the odor, there was strong phase locking of local field potentials (LFPs) between auditory and olfactory cortices (Zhou et al., 2019).

As auditory or visual stimuli may cause effective predictive projections to olfactory cortex, facilitating crosssensory integration and odor identification, and as this ability appears especially vulnerable to effects of common neuropathological disorders, research on the physiologic mechanisms underlying this task is warranted. We developed a novel auditory-odor association task in rats, wherein the animal must learn that a specific auditory tone, when associated with a specific odor, predicts reward (Go signal), whereas the same tone associated with a different odor, or vice versa, does not (No-Go signal). This design may be characterized as a simple odor identification task, as one odor needs to be matched to the correct non-olfactory cue. Because of the slight lag between tones and odors, this design also allowed analyses of separate auditory and olfactory driven activity within the olfactory and auditory cortices. We explored the effects of learning on multisensory-evoked activity within auditory and olfactory cortex, as well as the effects of context on this activity. Together, the results demonstrate the importance of both learning and context in expression of multisensory cortical processing in olfaction.

\section{Materials and Methods}

\section{Subjects}

Male Long Evans hooded rats $(n=15 ; 200-450 \mathrm{~g})$ from Envigo Lab Animals were used as subjects. Animals were single housed with enrichment and had ad libitum access to food. Access to water was restricted during the training session and for 30 min 2-4 h after training while in the home cage. Body weight and health status were monitored daily and no rat declined under $85 \%$ of its initial weight over the course of training. Lights were on from 6 A.M. to 6 P.M. and all testing occurred in the light portion of the day. All handling, housing and experimental procedures were approved by, and performed in accordance with the Institutional Animal Care and Use Committee guidelines as well as National Institutes of Health guidelines for the proper treatment of animals. The animals in 
this experiment are referred to as odor-tone (OT)1, OT2, etc., when data from individual animals are presented.

\section{Behavioral assay}

Go/No-Go training occurred in a black Plexiglas operant chamber $(27 \times 27 \times 33 \mathrm{~cm}$; width $\times$ length $\times$ height $)$ with an infrared (IR) beam-monitored nose port on the back wall and an IR beam-controlled water port on a side wall. Breaking the beam in the nose port initiated a trial with a $90-\mathrm{dB}$ sine wave tone $(0.1$ or $1 \mathrm{kHz})$ lasting $500 \mathrm{~ms}$. Immediately after the end of the tone, an odor (peppermint or vanilla, McCormick) was delivered through the same nose port, and was maintained until the animal left the port. If the rat left the port $<500 \mathrm{~ms}$ following the tone onset, the trial was considered aborted. The animals were required to learn that a specific combination of tone and odor was the stimulus that signaled an available water reward (100 $\mu \mathrm{l}$; Go signal), if the animal broke the IR beam in the water port within $3 \mathrm{~s}$. Other combinations of tone and odor were not rewarded (No-Go signals). Following initial shaping, the probability of a Go trial was $60 \%$. During training sessions, all animals were held in the testing room, and thus had passive exposure to the sound of the tones generated by other rats. During initial shaping, in the first few days, the minimal nose-poke duration was $200 \mathrm{~ms}$, Go trial combinations occurred at $80 \%$ of trials, and all nose-pokes led to water reward. As the rats learned the task, these initial task settings were changed gradually during the first $10 \mathrm{~d}$, according to a standardized protocol, such that the final settings were 500-ms poke, $60 \%$ Go trials, and no reward on No-Go trials.

\section{Electrophysiology}

To examine cortical processing of multisensory signals, a subset of animals (well-trained, $n=8$; naive $n=6$ ) were used for LFP recording from the auditory cortex (A1) and anterior piriform cortex (aPCX). In addition, four animals were not able to learn the multisensory task after surgery, data from these animals were not considered. Rats were implanted with electrodes (stainless steel, $127 \mu \mathrm{m}$ ) directed toward A1 $(3.5 \mathrm{~mm}$ posterior to bregma, $6.5 \mathrm{~mm}$ lateral, $5.0 \mathrm{~mm}$ ventral) and toward aPCX $(1.0 \mathrm{~mm}$ anterior to bregma, $4.5 \mathrm{~mm}$ lateral, $8.0 \mathrm{~mm}$ ventral) to record LFPs from these regions during behavioral training. The electrodes were attached to a telemetry devise implanted under the dorsal skin (F20-EET, DSI, Inc). Analog signals were transmitted to a receiver placed under the operant chamber or under the homecage, digitized at $5 \mathrm{kHz}$, and collected and analyzed using Spike2 software (Cambridge Electronic Design). Given that the tone and odor stimuli did not temporally overlap, LFP responses to both could be extracted independently. LFP activity during the tone (500-ms period) and odor (250-ms period) stimuli was analyzed with fast Fourier transform (FFT; $4.9-\mathrm{Hz}$ bins), as well as activity during periods when the animal was disengaged from the task (i.e., not self-initiating trials for $>30 \mathrm{~s}$ ) which served as baseline. In addition to stimulus evoked activity during the Go/No-Go task, LFPs were also recorded when the animals were in their homecage. For these recordings, the homecage was placed next to the operant chamber while another rat was in training and generating trials. In this condition, the tones were audible in the homecage at the same intensity $(90 \mathrm{~dB})$ as in the operant chamber. This allowed analyses of tone evoked activity in animals while in the context of their homecage. Baseline LFP activity in the homecage during periods of no tones was also quantified. Given the design of the operant chamber and the brief duration of the stimulus, odors are presumed to not penetrate the homecage. For analysis of evoked activity in both the operant chamber and in the home cage, LFP activity during stimulation was expressed as a ratio of baseline activity.

In addition to stimulus evoked activity, spectral coherence of the power of LFP oscillations between A1 and aPCX (9.7- $\mathrm{Hz}$ bins) was examined using the cohere script in Spike2. A1-aPCX coherence was measured in three different states. (1) During active sampling. Data were obtained from $1 \mathrm{~s}$ of data starting at trial onset for each of the first 100 trials, regardless of trial type, within a session. (2) When the animal was in the operant chamber but disengaged from the task ( $>30$-s period). For this analysis, coherence was determined across the full period of time (not trial dependent). (3) When the animal was in their home cage while another rat was in training and generating trials $(>30 \mathrm{~s}$ of tone-free activity, again not trial dependent).

An additional measure of functional connectivity was applied to more closely examine trial-related connectivity. To examine tone-evoked changes in phase locking between $\mathrm{A} 1$ and $\mathrm{aPCX}$ during trials, we calculated the phase lag index (PLI; Stam et al., 2007) across trials. PLI is a measure of phase synchrony with reduced bias from common sources and volume conduction (Stam et al., 2007). In brief, LFPs were bandpass filtered at log-spaced frequencies ranging from 1 to $30 \mathrm{~Hz}$ with a fixed bandwidth of $2 \mathrm{~Hz}$. Instantaneous phase time series were extracted for A1 and aPCX using the Hilbert transformation. The resulting between-region phase difference time series were segmented into epochs from $0.5 \mathrm{~s}$ before $2 \mathrm{~s}$ following tone onset. Then, PLI was calculated at each time-frequency point and baseline corrected by subtracting the baseline ( $0.5 \mathrm{~s}$ before tone onset) average. Finally, the statistical significance of the PLI change was evaluated using a permutation method. For each permutation, the trial indices for one channel (A1/aPCX) were shuffled and the permuted PLI was calculated. After repeating this procedure 5000 times, we obtained a distribution of permuted PLI values at each time-frequency point. The mean and standard deviation of this distribution was obtained by normal distribution fitting (MATLAB's normfit). A z score of the real PLI was then calculated by subtracting the mean of the distribution which was further divided by the standard deviation. Multiple comparison corrections were controlled using a cluster-based method. The $p$ value threshold for initial clustering was set at 0.001 (twosided, corresponding to a $z$ score of 3.29). The maximal cluster size (sum of absolute $z$ score) was retrieved for each permutation, resulting in a permuted cluster-size distribution. Corrected $p$ values of each cluster from the real PLI $z$ score map were calculated as the proportion of permuted clusters that were larger than the real one. 

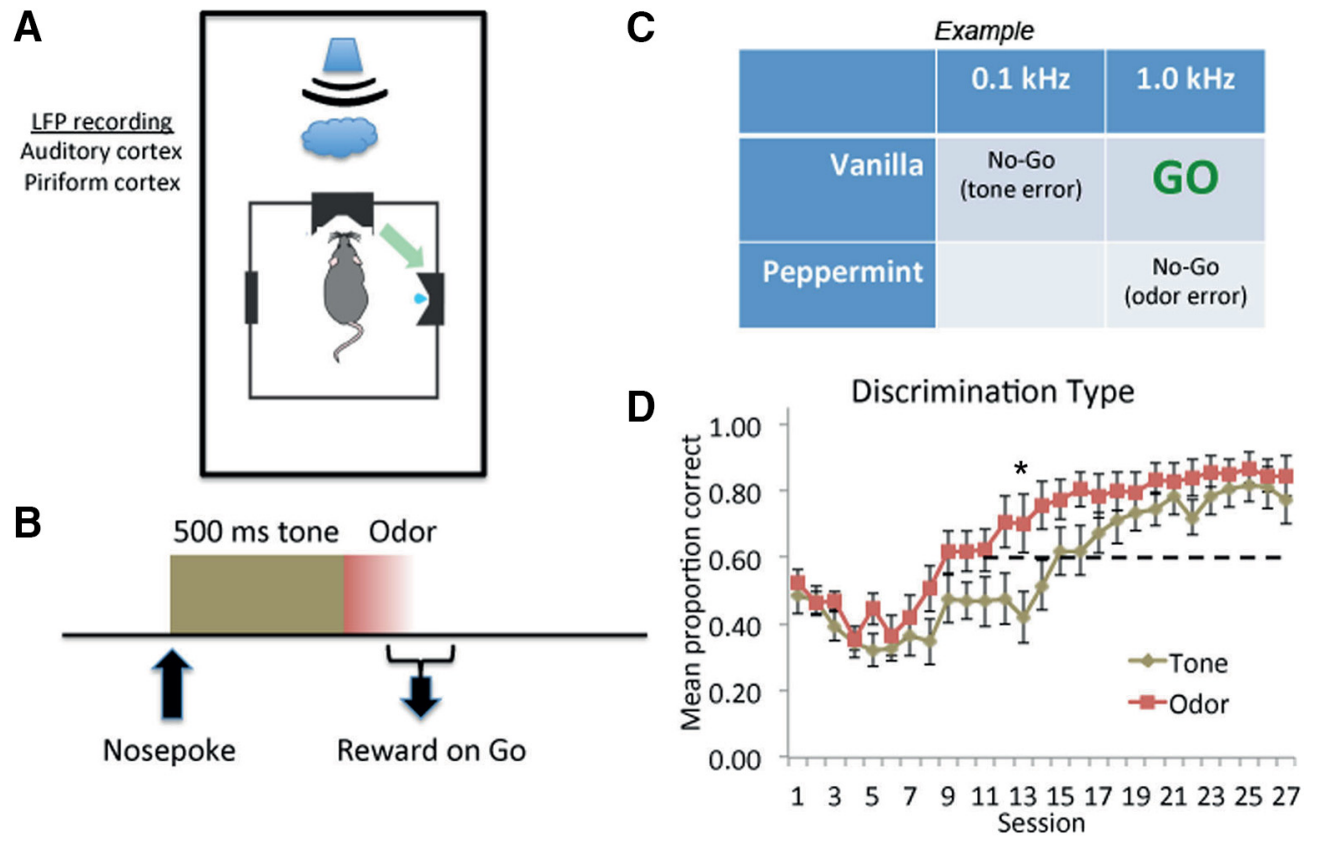

Figure 1. Experimental design and acquisition performance. $\boldsymbol{A}$, Rats initiated trials by nose poking a port which caused $(\boldsymbol{B})$ onset of one of two 500-ms, 90-dB tones. At tone offset, one of two odors were presented through the port. $\boldsymbol{C}$, Odors included vanilla and peppermint and the frequency of the tones were 0.1 and $1 \mathrm{kHz}$. Different tone-odor associations served as the Go stimulus in different animals, although in all cases successful identification of the Go stimulus required matching the correct tone with the correct odor. $\boldsymbol{D}$, Acquisition of correct performance, divided by error type (mean \pm SEM). Rats relatively quickly learned to reduce odor-based errors, but took longer to reduce tone-based errors. The horizontal dashed line represents chance performance. Initial decrease in performance is due to the fact that the task became progressively more difficult during the first $10 \mathrm{~d}$ of training (see Materials and Methods). The asterisk signifies a significant difference between Tone and Odor $(p<0.05)$.

We also compared the PLI change between Go and No-Go trials, and between training and homecage sessions using a cluster-based correction method. In each permutation, trials from both conditions were pooled together and each trial was assigned to one of the conditions randomly. Then, permuted PLI (baseline corrected) was calculated for each condition and its betweencondition difference was obtained. The real betweencondition difference was tested against the permuted difference in a similar was described above using the same initial $p$ value threshold (5000 permutations). To account for the difference in number of trials between different conditions, we estimated the PLI for the condition with a greater number of trials using a bootstrapping method. In each bootstrap, the PLI was calculated for a subset of trials (same number of the other condition) was randomly extracted from all trials. The above procedure was performed 200 times, and the average of all bootstraps was used as the final result.

\section{Results}

The experimental design and behavioral results are shown in Figure 1. Rats learned to associate an auditory tone of a specific frequency to a specific odor to receive a water reward (Go trials). As shown in Figure $1 D$, rats attained a mean of $80 \%$ correct, although performance acquisition varied based on error type. As shown in Figure $1 C$, there could be two error types on Go trials. If, for example, the Go stimulus was the association of the higher frequency and vanilla, then a Go response to the combination of high frequency and peppermint would be an odor-based error (correct frequency but wrong odor). If, in contrast, a Go response occurred to the lower frequency tone and vanilla, this corresponds to a tone-based error (correct odor but wrong frequency). In animals performing above chance on the task, the mean sampling duration was $688 \pm 20 \mathrm{~ms}$ (mean \pm SEM) on Go trials and $701 \pm 19 \mathrm{~ms}$ on No-Go trials. Trial type had no significant effect on sampling duration (Go trials, No-Go based on tone, No-Go based on odor, $\left.F_{(3,18)}=1.03, p=0.37\right)$. Thus, rats $(n=10)$ sampled the tone for the full $500 \mathrm{~ms}$ and the odor for nearly $200 \mathrm{~ms}$. While rats ultimately had similar tone- and odor-based performance by the end of training (Fig. 1D), they learned odor-based correct responses significantly faster over the course of training than tone-based responses as shown by the significant interaction in a stimulus $\times$ trial, repeated measures ANOVA $(2 \times 27$ repeated measures ANOVA: stimulus $\times$ trial interaction, $F_{(26,468)}=1.68, p=0.02$; main effect of trial, $F_{(1,26)}=37.24, p<0.0001$; main effect of stimulus, $\left.F_{(1,18)}=2.69, p=0.12\right)$.

To examine the effects of learning and context on oscillations in auditory and olfactory cortices, a subset of animals $(n=6)$ was implanted with electrodes before training onset, while the rest were implanted after they had attained criterion behavioral performance $(>70 \%$ correct; Fig. 2). Due to technical limitations, no animals were recorded throughout the entire five- to six-week training session. LFP activity during the tone sampling period and 
A

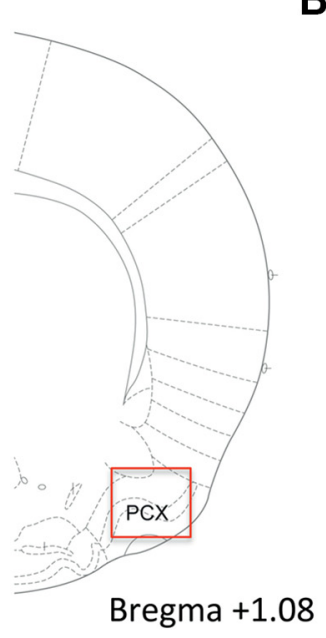

B

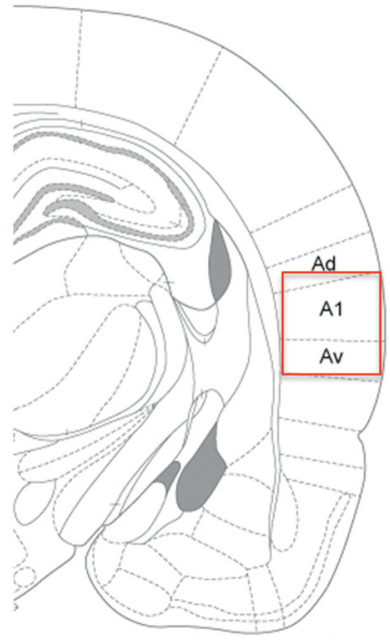

Bregma -3.48
C
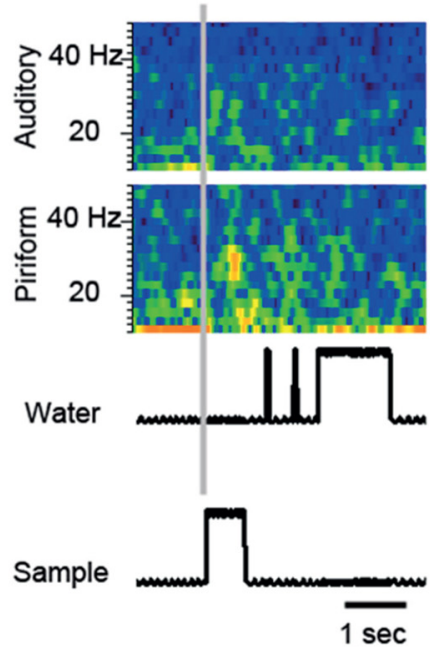

Figure 2. $\boldsymbol{A}$, Location of aPCX electrode. $\boldsymbol{B}$, Location of A1 electrode. $\boldsymbol{C}$, Representative pseudocolor spectrogram (blue $=$ low power, yellow $=$ high power) of stimulus evoked oscillatory responses in A1 and aPCX during trial initiation in a well-trained rat. IR beam activity for the odor sampling port (sample) and water reward port (water) are shown at bottom aligned with the simultaneously recorded spectrogram.

the odor sampling period were expressed as a ratio of baseline and separately analyzed in both A1 and aPCX. In addition to recording during the training session, responses to tones were also recorded while the animals were in their homecages. The homecages were placed next to the operant chamber during a training session for a different rat. The sound intensity was confirmed to be the same in the operant chamber and the homecage.

As shown in Figures 3, 4, in naive animals $(n=6)$ during initial two to three training sessions when performing at chance levels, small stimulus-evoked $\beta$ band $(15-35 \mathrm{~Hz})$ oscillatory responses were observed in aPCX and A1.
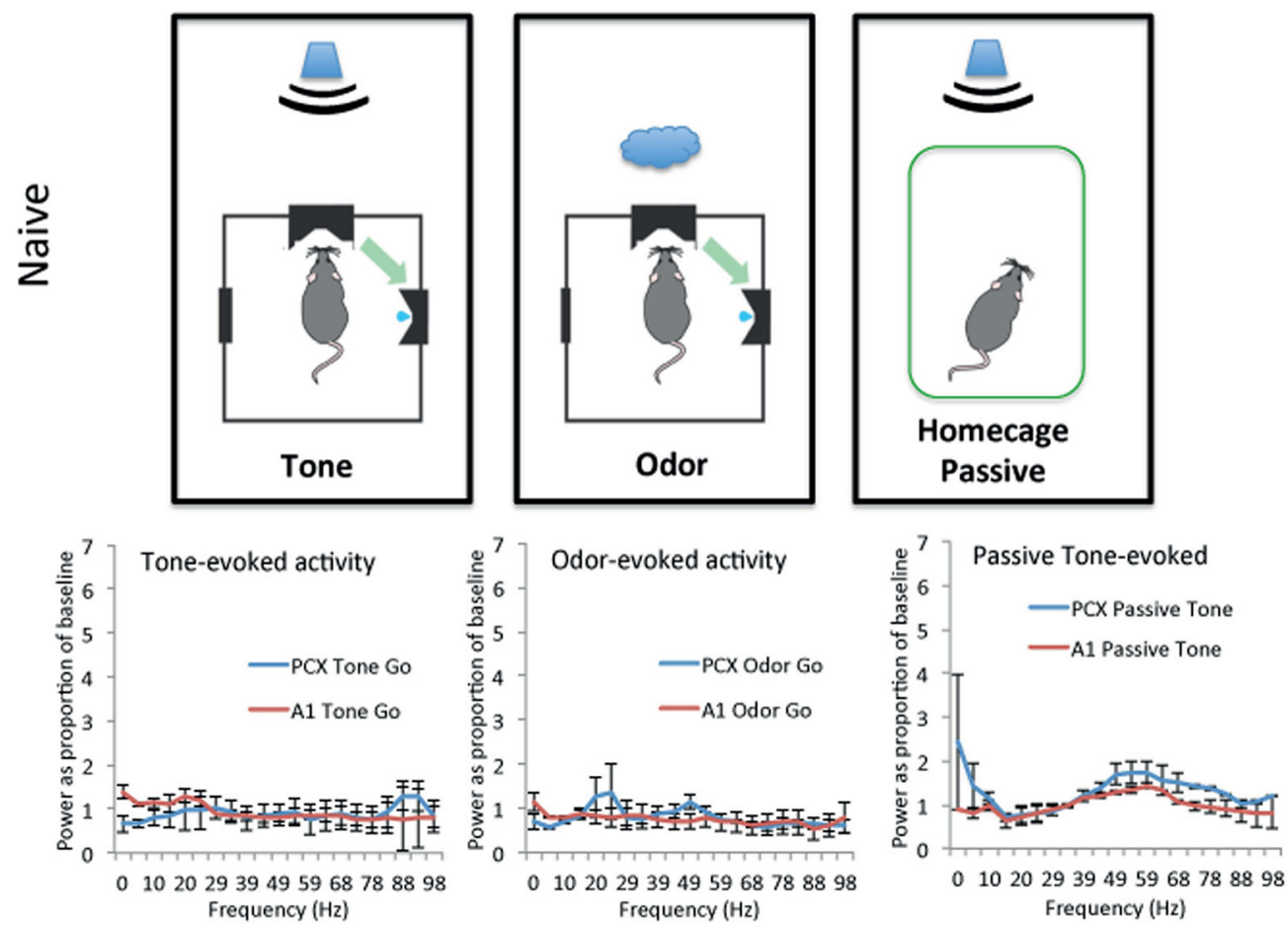

Figure 3. Stimulus evoked LFP activity in naive rats (during the initial two to three training sessions). LFP activity was assessed while the animals were actively initiating trials. Activity was quantified (1) during the $500 \mathrm{~ms}$ of tone presentation (tone-evoked); (2) for 250 $\mathrm{ms}$ starting at tone offset and odor onset (odor-evoked); and (3) during $500 \mathrm{~ms}$ of tone when the animals were in their home cage (passive tone-evoked). FFT analyses in each region to each stimulus were normalized as a ratio to baseline (disengagement from the task for at least $30 \mathrm{~s})$, data displayed as mean \pm SEM. 
A

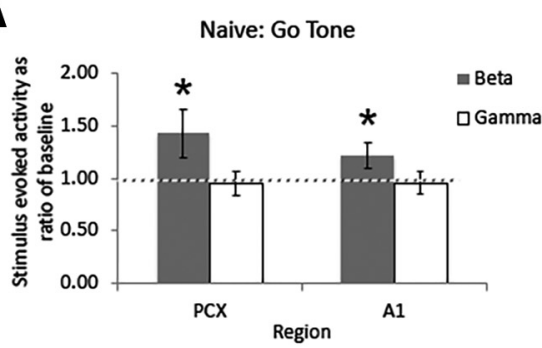

B

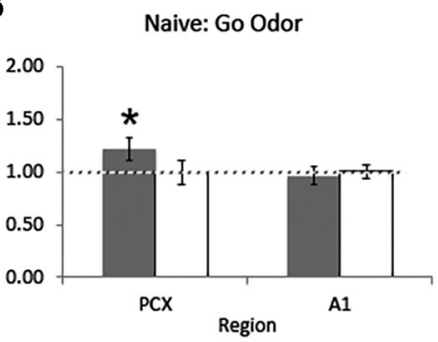

C

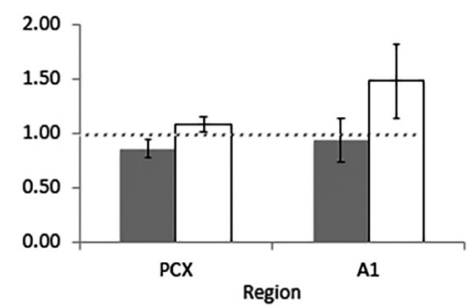

Figure 4. Quantification (mean \pm 1 SEM) of stimulus-evoked $\beta$ and $\gamma$ band LFP power in naive rats. $\boldsymbol{A}$, Responses to Go tone. $\boldsymbol{B}$, Responses to Go odor. Activity was quantified in the same way as in Figure 3. No significant evoked response was observed to either tone or odor during the task, although tone was associated with a significant $\gamma$ band response in aPCX. C, Responses to tone while rats were in their homecage. Asterisk signifies significant difference from 1, $p<0.05$ (PCX tone-evoked $\beta, p=0.044, \mathrm{~A} 1$ tone-evoked $\beta, p=0.047$, PCX odor-evoked $\beta, p=0.032$ ). The $\gamma$ response in A1 was not significantly different from 1.0.

Figure 3 displays the full FFT spectrum, while Figure 4 shows the same data collapsed across the $\beta$ and $\gamma$ frequency bands for statistical analyses. $\beta$ Band oscillations were significantly enhanced from baseline in both PCX and $\mathrm{A} 1$ in response to the tone, and in PCX in response to the odor (Fig. 3; one-sample $t$ tests vs a mean of 1 , df $=$ 5 , ps $<0.05)$. $\gamma$ Band $(35-90 \mathrm{~Hz}$ ) oscillations remained near baseline levels (Fig. 4).

In contrast to these very weak sensory evoked responses in naive rats, in animals trained to criterion (expert, $n=8$, with one animal having a failed aPCX electrode), robust multi-sensory (tones and odors) $\beta$ band oscillations were evoked in both aPCX and A1 (Fig. 5). In contrast, responses to tones presented while the animal was in their homecage were greatly reduced in both structures compared to during active training. No $\gamma$ band response above baseline was detected, thus statistical analyses were focused on $\beta$ band activity. As shown in Figure 6, both aPCX and A1 displayed significant evoked $\beta$ band activity in response to both tones and odors (significantly $>1$, one-sample $t$ tests, all $p s<$ 0.05). This was true for both tones that were a component of the Go signal and tones that were a component of the No-Go signal. The same tones presented while the animals were in their homecage, however, evoked no significant $\beta$ band activity in either aPCX or A1 (one-sample $t$ tests, $p>0.05$ ). Similar to tones, both aPCX and A1 showed significant $\beta$ band activity to Go
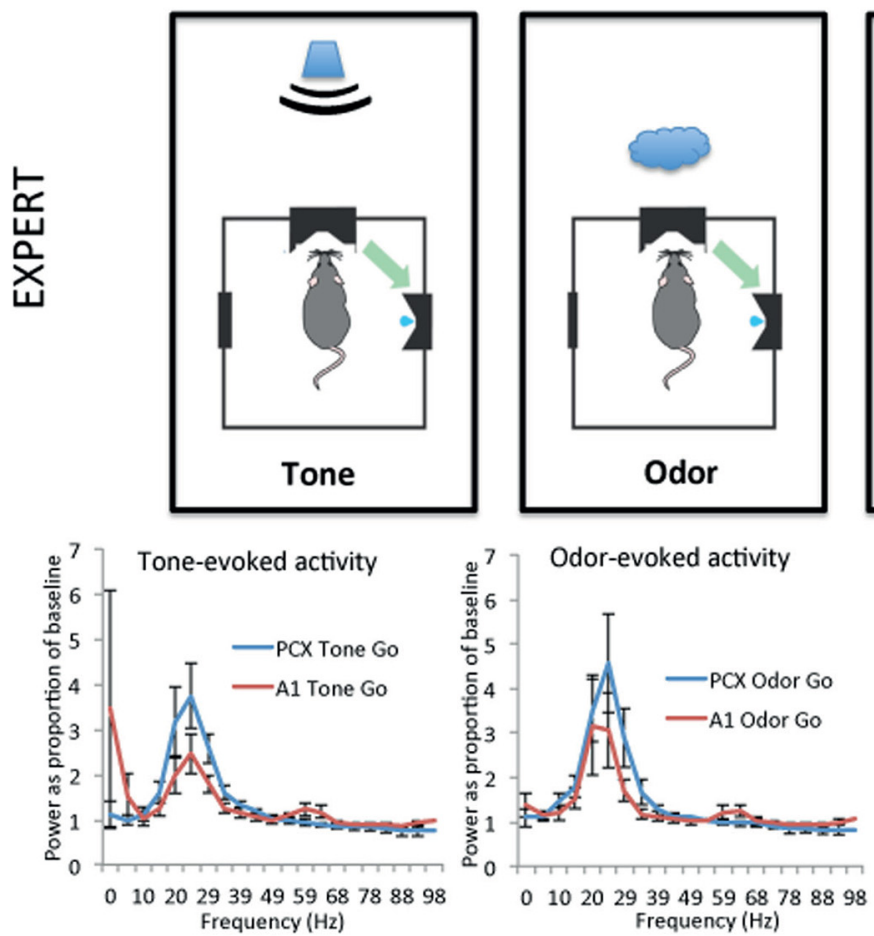
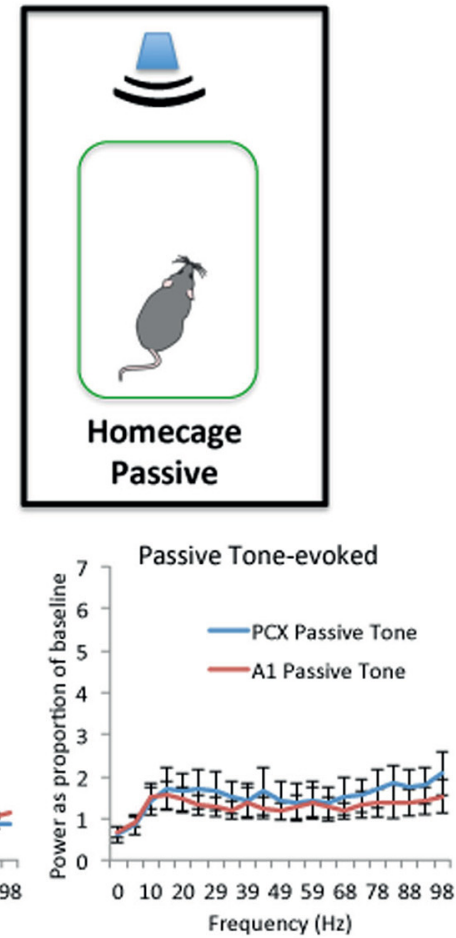

Figure 5. Stimulus evoked LFP activity in expert rats (mean \pm 1 SEM). Note the robust $\beta$ band activation in both aPCX and A1 in response to both the tone and odor while the animal was performing the task. This $\beta$ band response to the tone was reduced while the animal was in their home cage. No evoked $\gamma$ band activity was observed during training in either A1 or aPCX. FFTs show power as a percentage of baseline in $10-\mathrm{Hz}$ bins starting at the frequency displayed. 

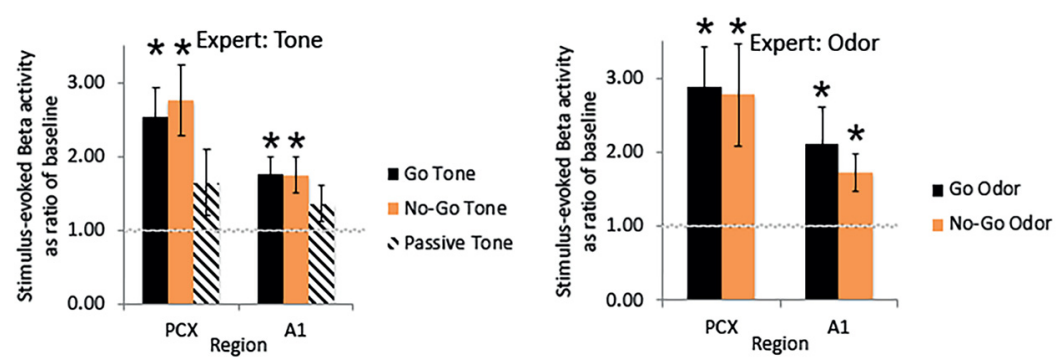

Figure 6. Quantification (mean \pm 1 SEM) of stimulus-evoked $\beta$ band LFP power to tone and odor in A1 and aPCX of Expert rats during the task and in the homecage. Both Go and No-Go tones and odors evoked significant $\beta$ band activity in the aPCX and A1, suggesting multisensory activation of these cortices. Presentation of the same tone while the animals were in their homecage, however, produced no significant $\beta$ band activity. Asterisks signify significant differences from $1, p<0.05$.

and to No-Go associated odors (Fig. 6). It should be noted that the animals were not asleep during the homecage stimulation, as assessed with the LFP (data not shown).

We next sought to determine if there was elevated functional connectivity between aPCX and A1 areas to indicate task- or context-dependent auditory-olfactory cortical communication. We compared the state-dependent multi-sensory activity in aPCX and A1 during training, relative to homecage activity $(n=7)$. aPCX-A1 LFP coherence was determined for three conditions: (1) active sampling; (2) disengagement from the task but while still in the operant chamber (defined as periods with no task activity of $>30 \mathrm{~s}$, animals were mostly grooming or exploring during this time); and (3) in the homecage while being exposed to tones generated during training by another rat. As shown in Figure 7, while rats were in the operant chamber, either actively engaged in the task or not engaged, aPCX-A1 coherence was significantly enhanced compared to being in the homecage (state $X$ frequency repeated measures ANOVA, main effect of state, $F_{(2,132)}=23.37, p<0.0001$; main effect of frequency, $F_{(10,132)}=3.65, p=0.0007$; post hoc Tukey tests revealed a significant difference between both engaged and disengaged rats from homecage rats at the 0 - to 10 and $10-$ to $20-\mathrm{Hz}$ frequency bins).

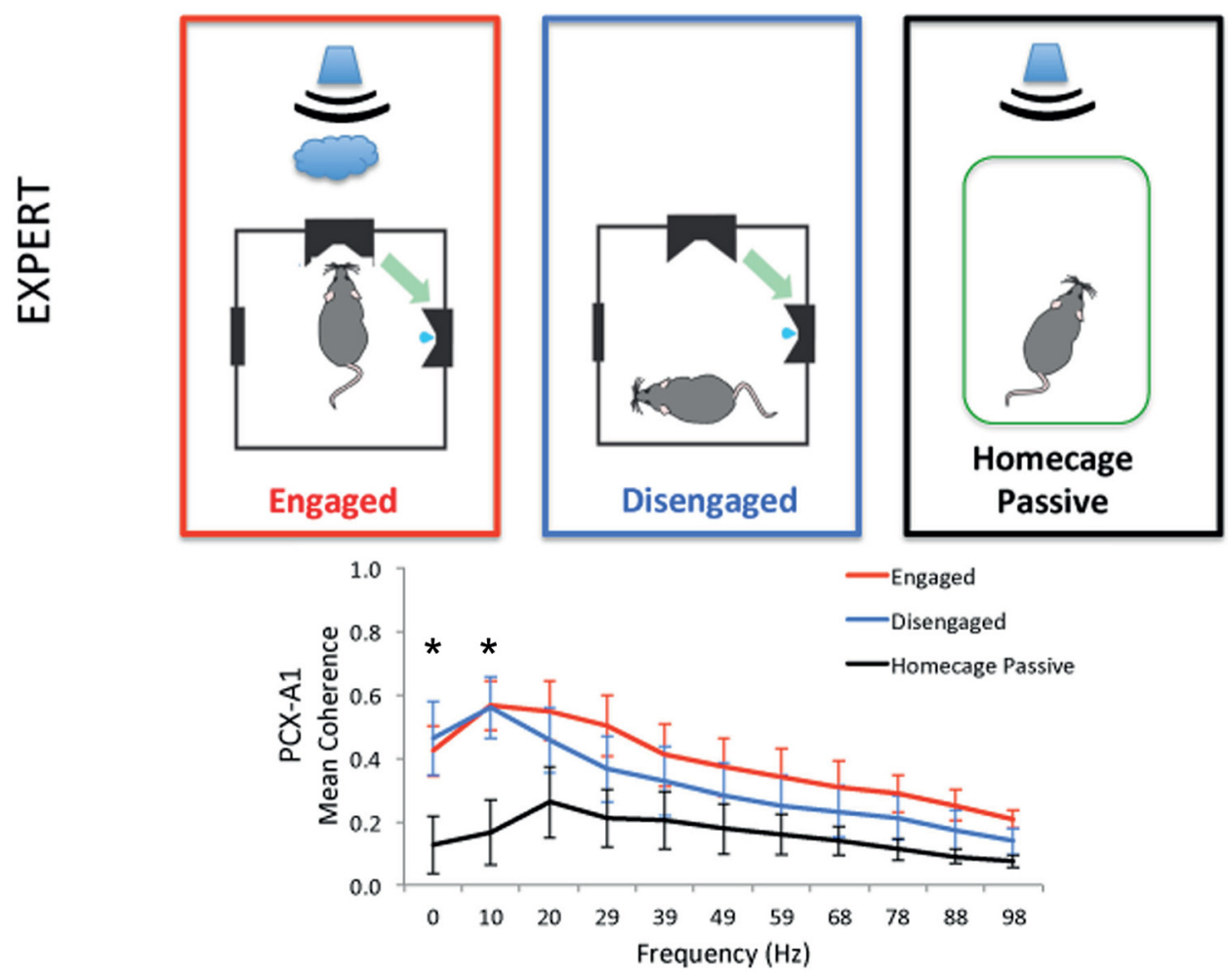

Figure 7. Spectral coherence between $\mathrm{A} 1$ and $\mathrm{APCX}$ is state-dependent in well trained rats (mean \pm 1 SEM). Graph shows coherence in $10-\mathrm{Hz}$ bins starting at the frequency displayed. Coherence is enhanced while the animals are actively engaged in the task and generating trials, and while the animals are in the operant chamber but not actively engaged in the task for at least $30 \mathrm{~s}$ compared to when the animals are in their homecage while another animal is in the operant chamber and generating trials. Asterisks signify significant differences from the homecage condition, $p<0.05$. 
OT1

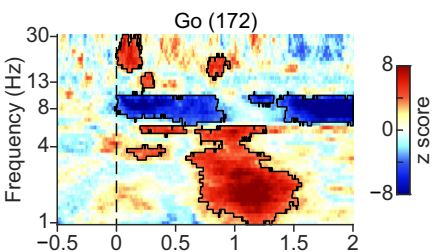

Go (184)

OT2

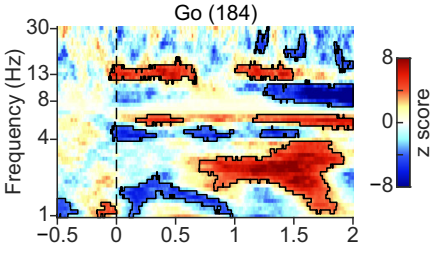

Go (108)

OT4

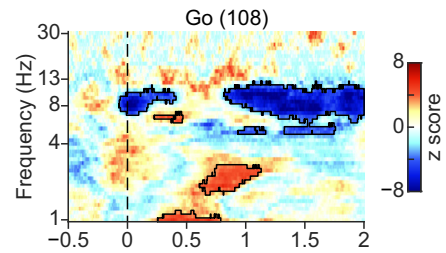

OT7

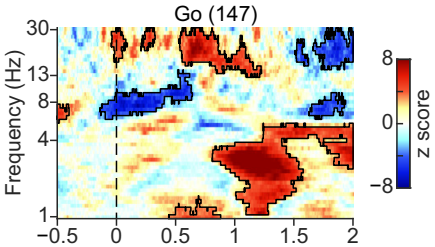

Go (55)

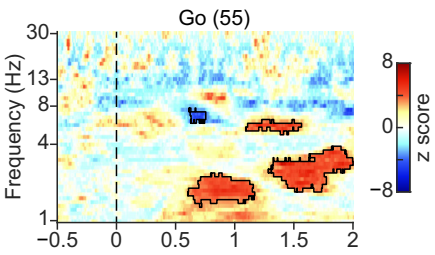

OT9

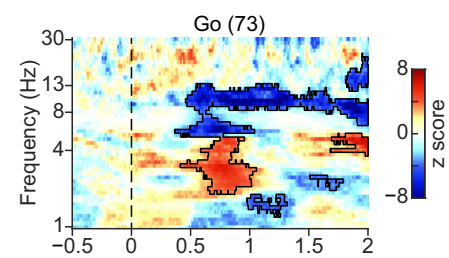

Go (134)

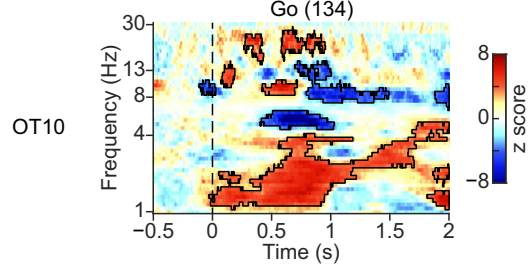

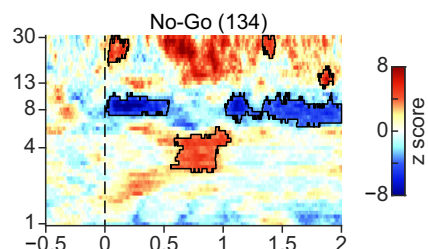

No-Go (136)
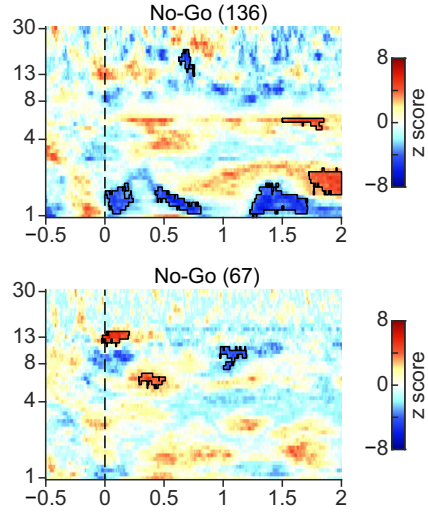

No-Go (99)
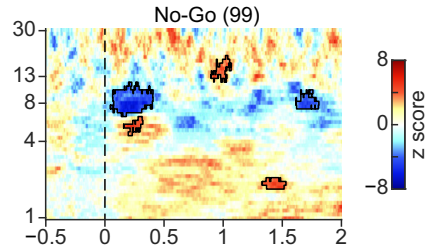

No-Go (45)
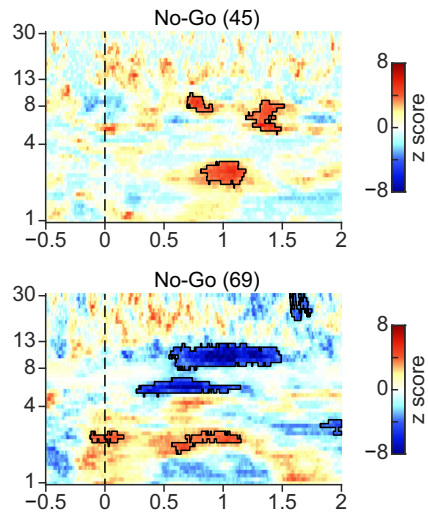

No-Go (35)
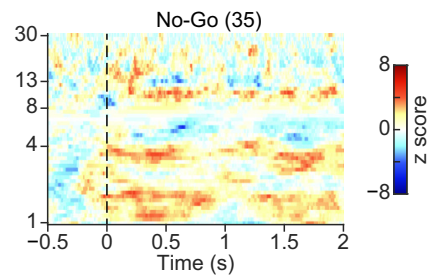

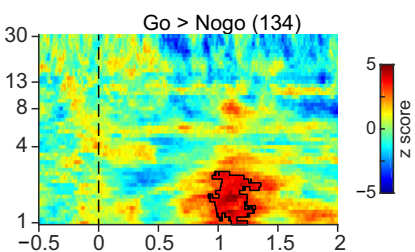

Go > Nogo (136)

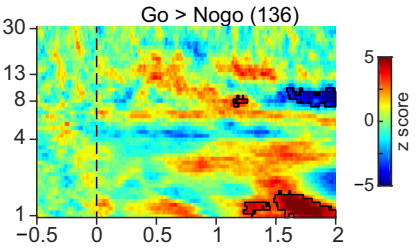

Go > Nogo (67)
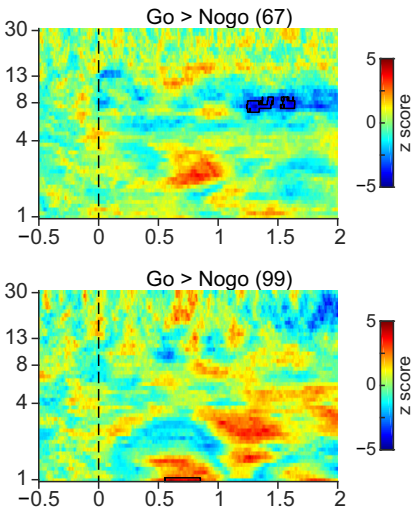

Go > Nogo (45)

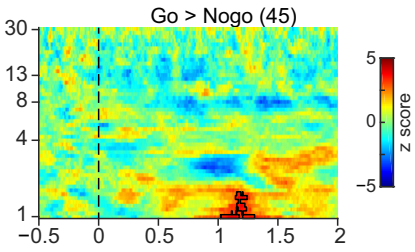

Go > Nogo (69)

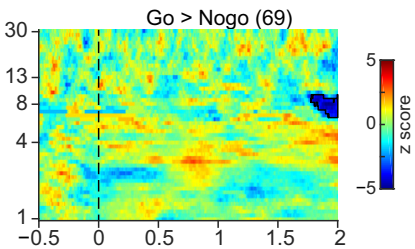

Go > Nogo (35)

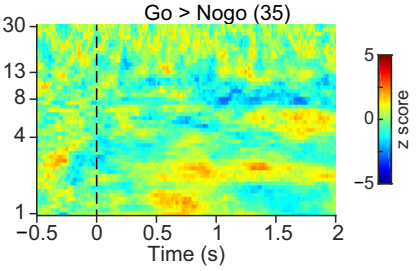

Figure 8. PLI analysis for seven individual rats for Go and No-Go trials, and the difference between trials. The PLI was baseline corrected $([-0.5,0] s$ before tone onset). The significance of the increase was tested using a cluster-based permutation test. The threshold for initial clustering was $p=0.001$. Outline regions indicate statistically significant clusters $(p<0.05)$. PLI increase difference between Go and No-Go trials. Most animals showed a significant increase in PLI beginning during the sampling period (sampling occurred 0-0.7 s) which extended into the reward period (1-2 s after trial onset). As shown in the right column, there was no consistent difference between Go and No-Go trials.

In addition to LFP coherence, we examined PLI between the LFPs recorded in A1 and aPCX in each rat. As can be seen in Figure 8, the PLI index (baseline corrected) between A1 and aPCX during Go trials was significantly elevated in a low frequency band $(<5 \mathrm{~Hz})$ during trials. In most animals, this enhanced PLI began near tone offset/ odor onset $(0.5 \mathrm{~s})$ and extended through water reward (1-1.5 s after trial onset). In most (5/7) animals this same pattern was observed during No-Go trials. However, a direct comparison between Go and No-Go trials (Fig. 8, 

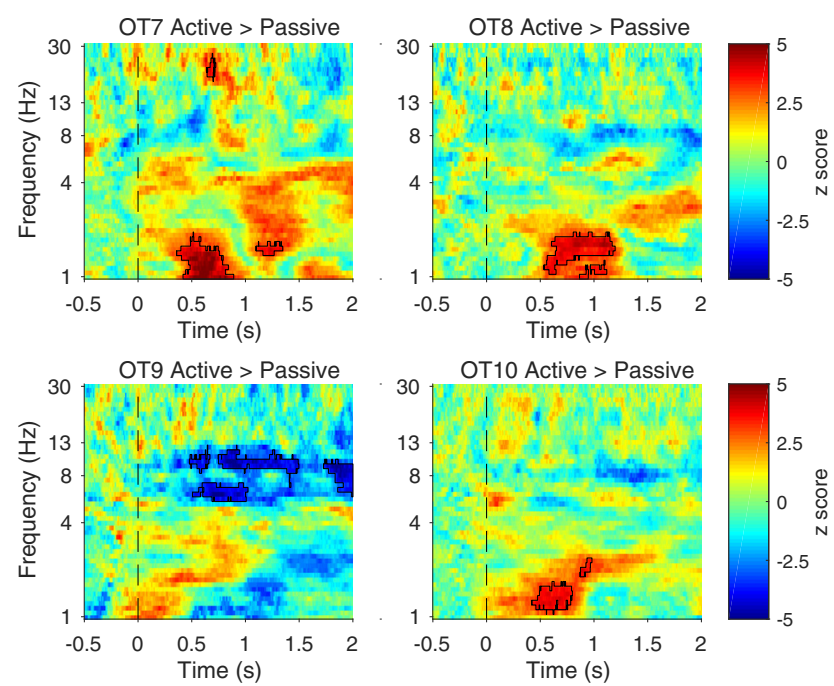

Figure 9. Tone induced stronger PLI increase while the animals were exposed to tones in the training session compared to the homecage. Data from animals tested in both conditions are shown as a difference between active and homecage conditions. The significance of the increase was tested using a clusterbased permutation test. The threshold for initial clustering was $p$ $=0.001$. Outlined regions indicate statistically significant clusters $(p<0.05)$.

right column) showed rats OT1, OT2, and OT8 had significantly higher PLI during Go trials, primarily after the sampling period which may be related to reward consumption.

We also analyzed A1-aPCX PLI in response to tones presented while the animals were in their homecage $(n=$ 4). Again, similar to our LFP coherence analyses, the tone-evoked A1-aPCX connectivity increase that occurred during active training was significantly reduced when the animals were stimulated in their homecage (Fig. 9). Note, however, that while a consistent pattern is observed across OT7, OT8, and OT10, OT9 does not show any reduction.

\section{Discussion}

Here, we describe a novel auditory-olfactory associative learning task that requires rats to learn to associate specific sounds with specific odors. The rats acquire this task relatively well, although show an initial bias toward odor cues. Within this task, rats demonstrate learningand context-dependent multisensory processing in aPCX and $A 1$. In well-trained rats, $A 1$ displayed a robust $\beta$ band oscillation in response to both learned tones and learned odors, as did the aPCX. These multisensory evoked responses were not observed in untrained rats. In addition to the multisensory evoked responses, functional coupling between aPCX and A1 was enhanced, as assessed with LFP coherence and phase locking. Importantly, both the evoked responses and the functional connectivity changes were only expressed while the rats were in the operant chamber, and not expressed when in their homecage. These results contribute to our understanding of multisensory processing effects in olfaction. Recognizing and identifying odors relies on effectively using pre- dictive cues from other senses (e.g., when being asked to smell milk to check whether it is spoiled, we predict what the milk will smell like before odor sampling; Zelano et al., 2011). Integration of matching non-olfactory and olfactory cues is assessed in common clinical olfactory assessments (Doty et al., 1984; Hummel et al., 2007), and thus understanding the neural processing underlying such behavioral performance is critical.

In expert animals, both tone and odor stimuli evoked $\beta$ oscillations in both A1 and aPCX. By definition, these appear to be multisensory evoked responses in classically unimodal cortices. In fact, links between the auditory and olfactory cortex in rodents have been well described (Budinger et al 2006; Budinger and Scheich 2009; Wesson and Wilson 2011). Furthermore, $\beta$ band oscillations are typically associated with inter-regional communication (Tallon-Baudry et al., 2001; Engel and Fries, 2010) including within the olfactory system (Kay, 2014), and are sensitive to learning, typically increasing with behavioral improvement (Martin et al., 2004, 2007; Kay, 2014). However alternative mechanisms of multi-sensory evoked responses in A1 and aPCX are possible. For example, responses need not be directly driven by sensory afferents to the cortex itself, but rather could reflect changes in activity due to very indirect or even neuromodulatory circuits. For example, the temporal structure of PCX unit activity is shaped by locus coeruleus activation, causing unit firing to be more strongly in phase with respiration (Bouret and Sara, 2002). Thus, activation of the locus coeruleus, for example, by an alerting stimulus like foot shock, could evoke a change in PCX activity that was not truly a somatosensory evoked response, but rather a more general response to a change in neuromodulatory tone.

A role for involvement of neuromodulatory inputs in shaping the observed multisensory responses is further supported by the fact that they were context-dependent. The same auditory stimulus that evoked robust $\beta$ band oscillations in A1 and APCX while the animal was engaged in the task evoked no such activity when the animal was in their homecage. The change in context could modify attention or alertness, which can directly modify sensoryevoked responses in the olfactory (Carlson et al., 2018) and other sensory systems (Fritz et al., 2007; Dykstra et al., 2017; Moore and Zirnsak, 2017). Thus, it is difficult to distinguish between changes in context or changes in state as the driver of these effects. Further work is necessary to explore the underlying mechanisms of these learned and context-dependent multisensory responses.

In addition to the learned multisensory-evoked responses, functional connectivity between A1 and aPCX was also enhanced in a learning- and context-dependent manner. This effect was apparent using either LFP coherence over long (100 s) periods of time while the animal engaged in the task, or in trial-related phase-locking analyses (PLI). Given that the learned change in A1-aPCX functional connectivity was context-dependent suggests that stable synaptic plasticity, such as long-term potentiation, is not a primary mechanism. Rather, the data suggest a flexibility in multisensory processing, where under 
specific conditions sensory systems are more likely to function coherently, while a change in conditions allows them isolate from each other. Similar context-dependent expression of learned changes in network connectivity have been previously reported within the olfactory cortex itself (Cohen et al., 2015; Cohen and Wilson, 2017).

While the changes in aPCX and A1 LFP sensory-evoked power were in the $\beta$ frequency band, the changes in functional connectivity were in lower frequencies $(\leq 10$ $\mathrm{Hz}$ ). This may suggest that the sensory-evoked $\beta$ band oscillations in aPCX and A1 are not imposed on the two structures by a third $\beta$ band driving region, but rather locally generated. However, enhanced functional connectivity at low frequencies could contribute to local high frequency activity via cross-frequency coupling in the two regions, although this requires further investigation (Hyafil et al., 2015). The mechanisms of the low frequency PLI coupling between A1 and aPCX during sampling/reward are not clear. Our LFP coherence measures peaked in a similar low frequency range but were present both while the animals were actively engaged in the task and when they specifically were not, and instead were generally grooming or exploring. This would argue that active sniffing (6-9 Hz; Kepecs et al., 2007; Wesson et al., 2008) may not be not a major generator of A1-PCX connectivity here, although we did not monitor respiration directly. Furthermore, in humans performing an auditory-word-cued odormatching task, high auditory-olfactory cortex coupling during the task was not respiration-dependent (Zhou et al., 2019). However, respiration has been shown to be important for linking activity within broad networks beyond the olfactory system in both humans (Zelano et al., 2016) and rats (Yanovsky et al., 2014; Lockmann et al., 2016; Moberly et al., 2018; Tort et al., 2018), and this respiration entrainment is important for odor memory and cognition (Zelano et al., 2016; Arshamian et al., 2018; Nakamura et al., 2018).

Finally, while odor detection and discrimination can be disrupted in a variety of disorders, odor identification, the linking of an odor sensation with a symbolic label, is generally most vulnerable to pathology associated with dementia (Murphy, 1999; Devanand et al., 2000) even in early and pre-clinical stages (Calhoun-Haney and Murphy, 2005; Olofsson et al., 2016; East et al., 2018). Similarly, animal models of dementia and dementia risk factors often show no deficits in odor discrimination (Vloeberghs et al., 2008; Phillips et al., 2011; Xu et al., 2014), despite impaired odor memory (Wesson et al., 2010) and olfactory system electrophysiological dysfunction (Wesson et al., 2011; East et al., 2018). More complex animal model tasks, such as the auditory-olfactory associative learning task characterized here, may more closely model multisensory links between olfaction and language that are so vulnerable in humans.

\section{References}

Arshamian A, Iravani B, Majid A, Lundstrom JN (2018) Respiration modulates olfactory memory consolidation in humans. J Neurosci 38:10286-10294.

Bekkers JM, Suzuki N (2013) Neurons and circuits for odor processing in the piriform cortex. Trends Neurosci 36:429-438.
Boisselier L, Gervasoni D, Garcia S, Ferry B, Gervais R (2018) Neuronal dynamics supporting formation and recombination of cross-modal olfactory-tactile association in the rat hippocampal formation. J Neurophysiol 119:1140-1152.

Bouret S, Sara SJ (2002) Locus coeruleus activation modulates firing rate and temporal organization of odour-induced single-cell responses in rat piriform cortex. Eur J Neurosci 16:2371-2382.

Budinger E, Scheich H (2009) Anatomical connections suitable for the direct processing of neuronal information of different modalities via the rodent primary auditory cortex. Hear Res 258:16-27.

Budinger E, Heil P, Hess A, Scheich H (2006) Multisensory processing via early cortical stages: connections of the primary auditory cortical field with other sensory systems. Neuroscience 143:10651083.

Cain WS (1979) To know with the nose: keys to odor identification. Science 203:467-470.

Calhoun-Haney R, Murphy C (2005) Apolipoprotein epsilon4 is associated with more rapid decline in odor identification than in odor threshold or dementia rating scale scores. Brain Cogn 58:178182.

Calvert GA, Brammer MJ, Bullmore ET, Campbell R, Iversen SD, David AS (1999) Response amplification in sensory-specific cortices during crossmodal binding. Neuroreport 10:2619-2623.

Carlson KS, Gadziola MA, Dauster ES, Wesson DW (2018) Selective attention controls olfactory decisions and the neural encoding of odors. Curr Biol 28:2195-2205.e4.

Choi I, Lee JY, Lee SH (2018) Bottom-up and top-down modulation of multisensory integration. Curr Opin Neurobiol 52:115-122.

Cohen L, Rothschild G, Mizrahi A (2011) Multisensory integration of natural odors and sounds in the auditory cortex. Neuron 72:357369.

Cohen Y, Wilson DA (2017) Task-correlated cortical asymmetry and intra- and inter-hemispheric separation. Sci Rep 7:14602.

Cohen Y, Putrino D, Wilson DA (2015) Dynamic cortical lateralization during olfactory discrimination learning. J Physiol 593:1701-1714.

Dalton P, Doolittle N, Nagata H, Breslin PA (2000) The merging of the senses: integration of subthreshold taste and smell. Nat Neurosci 3:431-432.

de Araujo IE, Rolls ET, Velazco MI, Margot C, Cayeux I (2005) Cognitive modulation of olfactory processing. Neuron 46:671-679.

Demattè ML, Sanabria D, Spence C (2009) Olfactory discrimination: when vision matters? Chem Senses 34:103-109.

Devanand DP, Liu X, Tabert MH, Pradhaban G, Cuasay K, Bell K, de Leon MJ, Doty RL, Stern Y, Pelton GH (2008) Combining Early Markers Strongly Predicts Conversion from Mild Cognitive Impairment to Alzheimer's Disease. Biological Psychiatry 15:871-891.

Devanand DP, Michaels-Marston KS, Liu X, Pelton GH, Padilla M, Marder K, Bell K, Stern Y, Mayeux R (2000) Olfactory deficits in patients with mild cognitive impairment predict Alzheimer's disease at follow-up. Am J Psychiatry 157:1399-1405.

Doty RL, Shaman P, Kimmelman CP, Dann MS (1984) University of Pennsylvania Smell Identification Test: a rapid quantitative olfactory function test for the clinic. Laryngoscope 94(2 Pt 1):176-178.

Dykstra AR, Cariani PA, Gutschalk A (2017) A roadmap for the study of conscious audition and its neural basis. Philos Trans $\mathrm{R}$ Soc Lond B Biol Sci 372:20160103.

East BS, Fleming G, Peng K, Olofsson JK, Levy E, Mathews PM, Wilson DA (2018) Human Apolipoprotein E genotype differentially affects olfactory behavior and sensory physiology in mice. Neuroscience 380:103-110.

Engel AK, Fries P (2010) Beta-band oscillations-signalling the status quo? Curr Opin Neurobiol 20:156-165.

Foxe JJ, Morocz IA, Murray MM, Higgins BA, Javitt DC, Schroeder CE (2000) Multisensory auditory-somatosensory interactions in early cortical processing revealed by high-density electrical mapping. Brain Res Cogn Brain Res 10:77-83.

Fritz JB, Elhilali M, David SV, Shamma SA (2007) Auditory attentionfocusing the searchlight on sound. Curr Opin Neurobiol 17:437455. 
Gottfried JA, Dolan RJ (2003) The nose smells what the eye sees: crossmodal visual facilitation of human olfactory perception. Neuron 39:375-386.

Hummel T, Kobal G, Gudziol H, Mackay-Sim A (2007) Normative data for the "Sniffin' Sticks" including tests of odor identification, odor discrimination, and olfactory thresholds: an upgrade based on a group of more than 3,000 subjects. Eur Arch Otorhinolaryngol 264:237-243.

Hyafil A, Giraud AL, Fontolan L, Gutkin B (2015) Neural CrossFrequency Coupling: Connecting Architectures, Mechanisms, and Functions. Trends Neurosci 38:725-740.

Kadohisa M, Rolls ET, Verhagen JV (2005) Neuronal representations of stimuli in the mouth: the primate insular taste cortex, orbitofrontal cortex and amygdala. Chem Senses 30:401-419.

Karunanayaka PR, Wilson DA, Vasavada M, Wang J, Martinez B, Tobia MJ, Kong L, Eslinger P, Yang QX (2015) Rapidly acquired multisensory association in the olfactory cortex. Brain Behav 5:e00390.

Kay LM (2014) Circuit oscillations in odor perception and memory. Prog Brain Res 208:223-251.

Kepecs A, Uchida N, Mainen ZF (2007) Rapid and precise control of sniffing during olfactory discrimination in rats. J Neurophysiol 98:205-213.

Lockmann AL, Laplagne DA, Leão RN, Tort AB (2016) A respirationcoupled rhythm in the rat hippocampus independent of theta and slow oscillations. J Neurosci 36:5338-5352.

Maier JX, Blankenship ML, Li JX, Katz DB (2015) A multisensory network for olfactory processing. Curr Biol 25:2642-2650.

MacDonald SWS, Keller CJC, Brewster PWH, Dixon RA (2018) Contrasting olfaction, vision, and audition as predictors of cognitive change and impairment in non-demented older adults. Neuropsychology 32:450-460.

Mandairon N, Kermen F, Charpentier C, Sacquet J, Linster C, Didier A (2014) Context-driven activation of odor representations in the absence of olfactory stimuli in the olfactory bulb and piriform cortex. Front Behav Neurosci 8:138.

Martin C, Gervais R, Hugues E, Messaoudi B, Ravel N (2004) Learning modulation of odor-induced oscillatory responses in the rat olfactory bulb: a correlate of odor recognition? J Neurosci 24:389-397.

Martin C, Beshel J, Kay LM (2007) An olfacto-hippocampal network is dynamically involved in odor-discrimination learning. $J$ Neurophysiol 98:2196-2205.

McGurk H, MacDonald J (1976) Hearing lips and seeing voices. Nature 264:746-748.

Moberly AH, Schreck M, Bhattarai JP, Zweifel LS, Luo W, Ma M (2018) Olfactory inputs modulate respiration-related rhythmic activity in the prefrontal cortex and freezing behavior. Nat Commun 9:1528.

Moore T, Zirnsak M (2017) Neural mechanisms of selective visual attention. Annu Rev Psychol 68:47-72.

Morrot G, Brochet F, Dubourdieu D (2001) The color of odors. Brain Lang 79:309-320.

Murphy C (1999) Loss of olfactory function in dementing disease. Physiol Behav 66:177-182.

Nakamura NH, Fukunaga M, Oku Y (2018) Respiratory modulation of cognitive performance during the retrieval process. PLoS One 13:e0204021.

Neville KR, Haberly L (2004) Olfactory cortex. In: The synaptic organization of the brain, Ed 5 (Shepherd GM, ed), pp 415-454. New York: Oxford University Press.

Olofsson JK, Gottfried JA (2015) The muted sense: neurocognitive limitations of olfactory language. Trends Cogn Sci 19:314-321.

Olofsson JK, Rönnlund M, Nordin S, Nyberg L, Nilsson LG, Larsson M (2009) Odor identification deficit as a predictor of five-year global cognitive change: interactive effects with age and ApoEepsilon4. Behavior Genetics 39:496-503.

Olofsson JK, Rogalski E, Harrison T, Mesulam MM, Gottfried JA (2013) A cortical pathway to olfactory naming: evidence from primary progressive aphasia. Brain 136:1245-1259.

Olofsson JK, Josefsson M, Ekström I, Wilson D, Nyberg L, Nordin S, Nordin Adolfsson A, Adolfsson R, Nilsson LG, Larsson M (2016)
Long-term episodic memory decline is associated with olfactory deficits only in carriers of ApoE- $€ 4$. Neuropsychologia 85:1-9.

Phillips M, Boman E, Osterman H, Willhite D, Laska M (2011) Olfactory and visuospatial learning and memory performance in two strains of Alzheimer's disease model mice-a longitudinal study. PLoS One 6:e19567.

Schroeder CE, Mehta AD, Foxe JJ (2001) Determinants and mechanisms of attentional modulation of neural processing. Front Biosci 6:D672-D684.

Stam CJ, Nolte G, Daffertshofer A (2007) Phase lag index: assessment of functional connectivity from multi channel EEG and MEG with diminished bias from common sources. Hum Brain Mapp 28:1178-1193

Stevenson RJ, Mahmut MK (2011) Experience dependent changes in odour-viscosity perception. Acta Psychol (Amst) 136:60-66.

Stevenson RJ, Rich A, Russell A (2012) The nature and origin of cross-modal associations to odours. Perception 41:606-619.

Tallon-Baudry C, Bertrand O, Fischer C (2001) Oscillatory synchrony between human extrastriate areas during visual short-term memory maintenance. J Neurosci 21:RC177.

Tort ABL, Brankačk J, Draguhn A (2018) Respiration-entrained brain rhythms are global but often overlooked. Trends Neurosci 41:186197.

Veyrac A, Allerborn M, Gros A, Michon F, Raguet L, Kenney J, Godinot F, Thevenet M, Garcia S, Messaoudi B, Laroche S, Ravel $\mathrm{N}$ (2015) Memory of occasional events in rats: individual episodic memory profiles, flexibility, and neural substrate. J Neurosci 35 : 7575-7586.

Vloeberghs E, Van Dam D, Franck F, Serroyen J, Geert M, Staufenbiel M, De Deyn PP (2008) Altered ingestive behavior, weight changes, and intact olfactory sense in an APP overexpression model. Behav Neurosci 122:491-497.

Wesson DW, Wilson DA (2010) Smelling sounds: olfactory-auditory sensory convergence in the olfactory tubercle. J Neurosci 30: 3013-3021.

Wesson DW, Wilson DA (2011) Sniffing out the contributions of the olfactory tubercle to the sense of smell: hedonics, sensory integration, and more? Neurosci Biobehav Rev 35:655-668.

Wesson DW, Donahou TN, Johnson MO, Wachowiak M (2008) Sniffing behavior of mice during performance in odor-guided tasks. Chem Senses 33:581-596.

Wesson DW, Levy E, Nixon RA, Wilson DA (2010) Olfactory dysfunction correlates with amyloid-beta burden in an Alzheimer's disease mouse model. J Neurosci 30:505-514.

Wesson DW, Borkowski AH, Landreth GE, Nixon RA, Levy E, Wilson DA (2011) Sensory network dysfunction, behavioral impairments, and their reversibility in an Alzheimer's $\beta$-amyloidosis mouse model. J Neurosci 31:15962-15971.

Wilson DA, Sullivan RM (2011) Cortical processing of odor objects. Neuron 72:506-519.

Xu W, Lopez-Guzman M, Schoen C, Fitzgerald S, Lauer SL, Nixon RA, Levy E, Wilson DA (2014) Spared piriform cortical single-unit odor processing and odor discrimination in the Tg2576 mouse model of Alzheimer's disease. PLoS One 9:e106431.

Yanovsky Y, Ciatipis M, Draguhn A, Tort AB, Brankačk J (2014) Slow oscillations in the mouse hippocampus entrained by nasal respiration. J Neurosci 34:5949-5964.

Zelano C, Mohanty A, Gottfried JA (2011) Olfactory predictive codes and stimulus templates in piriform cortex. Neuron 72:178-194.

Zelano C, Jiang H, Zhou G, Arora N, Schuele S, Rosenow J, Gottfried JA (2016) Nasal respiration entrains human limbic oscillations and modulates cognitive function. J Neurosci 36:12448-12467.

Zellner DA, Kautz MA (1990) Color affects perceived odor intensity. J Exp Psychol Hum Percept Perform 16:391-397.

Zhou G, Arabkheradman G, Lane G, Gottfried JA, Schuele S, Rosenow J, Olofsson J, Wilson DA, Zelano C (2019) Human olfactoryauditory integration requires phase synchrony between sensory cortices. Nature Communications. Advance online publication. Retrieved March 11, 2019. doi: 10.1038/s41467-019-09091-3. 